Methods We asked patients attending Y Bwythn Newydd to complete a simple questionnaire about how they store and share information about their health in case of an emergency. We gave a Lions' tub to those patients that did not have one, explaining that the scheme provided information about their diagnosis, next of kin information, and any advanced care decisions such as preferred place of care/death. We taught patients how to use the 'emergency contacts' feature on their smartphone.

Results 31 patients completed the questionnaire. Only one of the patients was already using a MIAB. 28 MIAB tubs were handed out over four weeks. 29 out of 31 patients thought the scheme was a good idea. Nine out of 31 patients had a smart phone, of which four had set up the 'emergency contacts' feature.

Conclusions This project increased the use of the Message in a Bottle scheme, and the 'emergency contacts' smartphone feature. We have demonstrated how to increase the use of these methods of sharing emergency information. Advance care planning with simple interventions such helps empower patients and their families by ensuring their wishes are fulfilled at the end of life. More research is needed to further evaluate the impact of such interventions.

\section{THE DIFFICULTIES FACED BY FAMILY CAREGIVERS WHEN HOME IS THE PREFERRED PLACE OF DEATH}

Lynn Sudbury-Riley, Philippa Hunter-Jones, Ahmed Al-Abdin, Daniel Lewin, Sarah Dale, Karyn Johnston. University of Liverpool, East Cheshire Hospice

\subsection{6/spcare-2020-PCC.26}

Background Eighty per cent of people in the UK state they would prefer to die at home. A 'good death' is a key objective of palliative care and a comfortable death improves wellbeing among the bereaved. This study was designed to take a deepdive into the service experiences of a new Hospice@Home service, with the overall aim of ascertaining the human stories behind Hospice@Home helping people to achieve their preferred place of death.

Methods We collected 45 narratives from bereaved caregivers whose family member had received Hospice@Home in the weeks prior to their death. We used the Trajectory Touchpoint Technique (TTT), a rich pictures methodology that enables the collection of deep and rich data. The TTT encourages people to talk about their whole Hospice@Home journey in 5 stages: pre-arrival, arrival, clinical care and support, comfort, discharge and aftercare. Narratives were recorded and transcribed verbatim before being subjected to thematic analysis.

Results We found multiple problems. A lack of awareness and understanding of hospice help among both families and some referring clinicians delays access. Even when offered, resistance comes from a reluctance to allow 'strangers' into their homes, a hesitancy to hand over the care of their loved ones, and/or a disinclination to use the service due to not feeling entitled. These barriers persist despite many families being in crisis. Certainly, we found Hospice@Home positively impacts quality of life and quality of death for patients. However, families were unable to equate their experiences with a positive impact on their own quality of life, resulting in many re-thinking their own place of death preferences.
Conclusions There are major gaps in care provision, particularly notable in the wider healthcare system, including GPs, Pharmacies, access to equipment, and private care providers.

\section{Dementia | poster 6}

\section{MAPPING THE TRAJECTORY OF THE LAST 12 MONTHS OF LIFE OF PEOPLE WITH DEMENTIA: THE ROLE OF SPECIALIST PALLIATIVE CARE}

Jennifer Todd, Liz Reed. Princess Alice Hospice

\subsection{6/spcare-2020-PCC.27}

Background Dementia is now one of the top five underlying causes of death. There are concerns about the quality of care and barriers to the provision of quality end of life care for people with dementia, meaning they may not have access to specialist palliative care (SPC) or adequate symptom control.

Aim To map the last 12 months of life for people with dementia; the role of SPC

Method Retrospective, mixed method study reviewing clinical records of decedent patients and narrative interviews of the carer/next of kin.

Results Of 34 carers approached, 15 consented to being interviewed (44\% response).

2 typical trajectories were identified: 'A revolving door' recurrent acute events and crises, with regular attendance at accident and emergency (A\&E) and changes in care setting; and 'gentle fading', minimal health or social care intervention required).

13 patients had at least 1 visit to the A\&E department (range 1-6) but recurrent attendance was common. There was limited evidence of ACP and DNACPR was predominantly completed in the final days of life.

12 of the patients were referred to SPC. Median time from referral to death was 10 days: input included non-complex end of life symptom control, ACP, education and support of care home staff and family support.

Place of death was care home (5), hospital (6), hospice (2) and home (2). 7 died in their usual place of care.

Conclusion SPC has a role in the last 12 months of life for people with dementia but late referral limits the efficacy of SPC intervention. Local initiatives are needed for health and social care to work together with SPC to look at optimising care for people with dementia earlier in the illness trajectory.

\section{Education and training | posters $7-45$}

\section{OUTSIDE THE MARGINS: PALLIATIVE AND END-OF-LIFE CARE EDUCATION FOR HOMELESS SUPPORT SERVICES}

Gemma L Allen. Mary Stevens Hospice

\subsection{6/spcare-2020-PCC.28}

Background The CQC report 'A different ending: addressing inequalities in end of life care' highlighted homeless people with end of life care needs experience considerable barriers accessing palliative care. Mary Stevens Hospice developed a workshop for hostel workers, West Midlands Police, and Dudley Metropolitan, focused on palliative and end of life 